been referred to secondary care with two thirds being investigated or managed in primary care.

iLFT has increased liver disease diagnosis, improved quality of care and reduced unnecessary secondary care referrals.

\section{OTU-23 INCREASING ATTENDANCE AND ENGAGEMENT OF SUBSTANCE MISUSE CLIENTS WITH HEPATITIS C}

${ }^{1,2}{ }^{2}$ Clare Phillips*, ${ }^{2}$ Abdulkani Yusuf. ${ }^{1}$ Brighton and Sussex University Hospitals NHS Trust, Brighton, UK; ${ }^{2}$ East Sussex Healthcare NHS Trust, Eastbourne, UK

\subsection{6/gutjnl-2019-BSGAbstracts.428}

Introduction In June 2017, a hepatitis C (HCV) clinic was established in the local substance misuse service (SMS) as an outreach from the local district general hospital. The clinic initially operated one afternoon a month, but due to demand was increased to fortnightly in March 2018. The objective of the clinic was to provide HCV care (including work-up and treatment) to clients, who, because of their substance use, might not attend hospital clinics. The aim of this study was to review whether the SMS clinic improved attendance and engagement among these clients compared to the hospital setting.

Methods The outreach clinic database was used to identify all individuals who were offered an appointment in the outreach clinic between June 2017 and December 2018. The hospital electronic booking system was then used to review all historic appointments booked for each of these patients at the hospital. Attendance and engagement with the hospital clinic and the outreach clinic were compared.

Results 51 separate individuals were referred to the SMS clinic between June 2017 and December 2018. 31 of these (61\%) had, at some point (dating as far back as the early 2000s), been referred to the hospital gastroenterology service for their HCV. Of those referred, 12 (39\%) had never attended an appointment. $18(58 \%)$ attended at least once but were subsequently lost to follow-up. 1 (3\%) patient was started on HCV treatment at the hospital but transferred to the SMS clinic once it was established. By comparison, of the 51 referred to the SMS clinic, 17 (33\%) never attended an appointment, 12 (24\%) came at least once but were then lost to follow-up and $22(43 \%)$ remain engaged with the SMS clinic. Of the 22 engaged in the service; 5 have places to start treatment in the next few weeks, 7 have completed treatment and 4 are currently on treatment. 1 patient, lost to follow-up in the SMS clinic, was later treated in prison.

Conclusions Our study shows that, compared to a hospital setting, an outreach clinic at an SMS substantially improves attendance and engagement with care in this cohort of clients with HCV infection. There are a number of factors that may have contributed to the increase in attendance and engagement with the SMS clinic. SMS-based clinics have been shown to reduce barriers to HCV care for SMS attendees (Harris, 2017). We also believe that as our SMS service is based in the centre of town it makes it more accessible for clients compared to the local hospital which is on the peripheries of the town. Finally, an increased awareness of the new, highly effective direct acting antiviral (DAAs) for treatment of HCV might have improved acceptance of testing and treatment in this cohort.

\section{OTU-24 APPETITE FOR IMPROVEMENT; 50\% REDUCTION IN BLOODSTREAM INFECTIONS IN CENTRAL VENOUS CATHETER DELIVERED PARENTERAL NUTRITION}

Margaret Collins*, AJ di Mambro. Gloucestershire Hospitals NHSFT, Cheltenham and Gloucester, UK

\subsection{6/gutjnl-2019-BSGAbstracts.429}

Introduction Bloodstream infection (BSI) is a recognised risk with parenteral nutrition (PN) administered via a central venous catheter $(\mathrm{CVC})^{1}$. It can be life-threatening, leads to an extended hospital stay and, for patients reliant on PN, can mean -4 days without nutrition. NICE estimate the cost of each catheter-related BSI is $£ 9,900^{2}$.

Methods Quality improvement methodologies and tools were used to identify the problem, plan and test change ideas and measure improvements. BSI rates were recorded per 1000 days of PN to allow fair comparison of monthly BSI rates. The amount of PN administered per month was $8-16$ days. A root cause analysis tool (RCA) was developed to investigate each BSI thought related to a CVC. RCA outcomes were reported to the relevant ward manager to highlight areas for improvement and guide action planning. Findings from the RCAs were plotted on a Pareto chart to identify the most frequently occurring factors on which to focus improvements.

A driver diagram was used to plan the improvement process and identify change ideas. Engaging ward nurses, improving their knowledge and understanding of risk factors for developing a BSI and promoting best practice in management of CVCs were key aspects of this project.

1) Actions: Pop-up ward based teaching for nursing staff to highlight the risk factors for BSI and clarify best practice for management of CVC and PN. Nurses completed an anonymous questionnaire to gauge knowledge of $\mathrm{PN}$ and care of a CVC. Their responses guided teaching topics and content of posters to highlight best practice in CVC care.

2) Switch to single, rather than dual, lumen peripherally inserted central catheters (PICCs) to reduce the number of times the PICC was accessed.

3) For in patients requiring $\mathrm{PN}$ for more than 28 days, we trialled the use of a protective cleaning cap on the CVC lumen3.

4) Nutrition support team involvement in mandatory teaching sessions for F1 training and in Trust induction for safe CVC access in $\mathrm{PN}$ patients were also implemented.

Results A 50\% reduction in BSI rates in 2018 compared to 2017.

Conclusions RCA findings show that a number of factors contributed to our patients developing a BSI. Various strategies were used to improve BSI rates. The use of quality improvement tools and methodologies in all stages of this project was key to its success. Future work - develop a PN e-learning module - ward-based CVC update training This quality improvement project has focused on BSI in patients receiving PN however it could be applied to improving the care and use of all CVCs in our Trust. (1) Pironi L, Arends J, Bozzetti F, Cuerda C, Gillanders L, Jeppesen PB, Jolly F, Kelly D, Lal S, Staun M, Szczepanek K, Van Gossum A, Wanten G, Schneider SM, ESPEN Guidelines on chronic intestinal failure in adults. Clinical Nutrition. 2016;35(2): 24-07. (2) NICE. 
The 3M Tegaderm CHG IV securement dressing for central venous and arterial catheter insertion sites. Available from: https:/www.nice.org.uk/guidance/mtg25/chapter/5-Cost-considerations [Accessed 13th February 2019].

(3) NICE. Curos disinfecting cap for needleless connectors. Available from: https://www.nice.org.uk/advice/mib143/chapter/ The-technology [Accessed 13th February 2019].

\section{OTU-28 DON'T ASK, WON'T TELL: IMPROVING ALCOHOL SCREENING AND HOSPITAL ALCOHOL TEAM REFERRALS IN A\&E}

Graham Robinson*, Mahesh Bhalme, Veronica Hall, Gemma Heyes, Langlands Gillian, Liggett Nicola, Samantha Osborne. Royal Bolton Hospital, Bolton, UK

\subsection{6/gutjnl-2019-BSGAbstracts.430}

Introduction This Hospital's Alcohol Team provides a 7-day service, cited in the 10 Year NHS England Plan as having improved the quality of alcohol-related care. The Alcohol Team receive over 1700 referrals per year - predominantly from assessment wards where screening for potential alcohol issues of new admissions is mandatory and routine. Alcohol screening had not traditionally been routine for A\&E patients. There were concerns that the introduction of mandatory screening might risk complicating patient assessment, treatment and timely discharge. Initiatives were introduced to increase the incidence of screening in A\&E. An analysis was undertaken on the impact of these initiatives on referrals and patients.

Methods

Initiatives introduced in 2018 1) Partnership working between A\&E and Hospital Alcohol Team;

- Development of an Alcohol Assessment sticker, for optional incorporation into patient $A \& E$ notes, to facilitate the identification and management of potential alcohol issues ;

- Training targeted to A\&E Nurses;

- A\&E Link Nurse developed promotional displays and acted as a champion in A\&E to raise awareness.

Referral data was audited to assess the impact of the initiatives on referrals. A $10 \%$ randomised sample of referrals was further analysed to assess the impact of the increase in referrals. Case Studies were captured to illustrate the benefits of earlier identification of alcohol issues.

Results There was a $16 \%$ increase (240 patients) in total referrals received: 1523 (2017) to 1763 (2018). A\&E referrals rose $79 \%$ from 217 to 389 . This is against a back-drop of the rate of hospital admissions attributed to alcohol, nationally and locally, remaining broadly flat. 59\% of patients referred to the Alcohol team were discharged from A\&E (compared to $55 \%$ in 2017); the average length of stay of those discharged patients was 5.6 hours (compared to 6.0 hours in 2017), and $48 \%$ of these patients were discharged in under four hours (compared to $42 \%$ the previous year). Specific cases were captured in case studies to illustrate the mechanisms through which early identification of potential alcohol issues can lead to better management of alcohol-related issues, facilitate safe discharge and reduce length of stay.

Conclusions The screening, education, and partnership initiatives led to an increase in referrals from $A \& E$ to the hospital alcohol team. An audit of referrals suggests the earlier identification of alcohol-related issues in A\&E has not had negative impacts on discharges and length of stay from A\&E. In practice the Alcohol Team has found that earlier identification of potential issues has enabled them to intervene earlier to improve the management and timely discharge of patients.

\section{Abstracts of Distinction}

\section{ATU-10 TARGETING HISTOPATHOLOGY WORKLOAD CRISIS USING ATTENUATED TOTAL REFLECTION FOURIER TRANSFORM INFRARED (ATR-FTIR) SPECTROSCOPY}

Ilaria Idini* ${ }^{*}$ Caroline Ball, Katherine Willetts, Lance Farr, Caitlin Langford, Liberty Foreman. BeamLine Diagnostics trading as DynamX Medical, Oxford, UK

\subsection{6/gutjnl-2019-BSGAbstracts.431}

Introduction For the past 200 years histopathology has been the gold-standard for identification of many diseases. Biopsy procedures typically generate $1-0$ sample sections for analysis,

\section{Complete DynamX System}

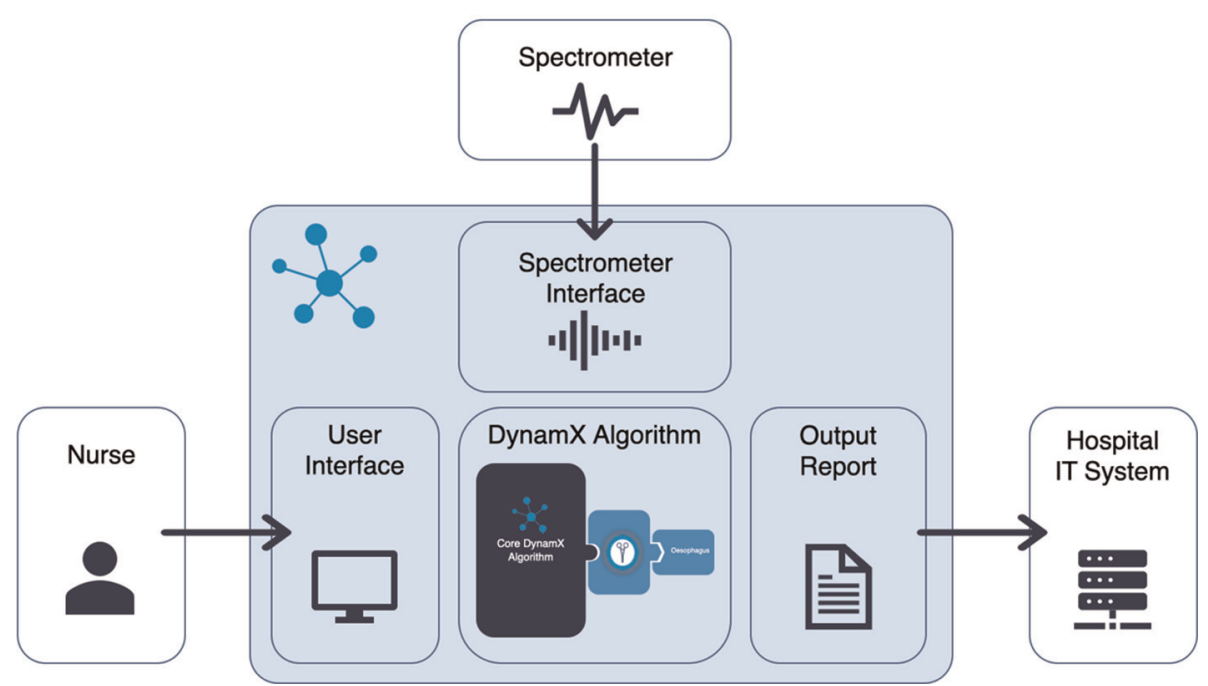

